\title{
Durum wheat (Triticum durum Desf.) Mediterranean landraces as sources of variability for allelic combinations at $G l u-1 / G l u-3$ loci affecting gluten strength and pasta cooking quality
}

\author{
Ruyman Nazco $\cdot$ Roberto Javier Peña \\ Karim Ammar · Dolors Villegas · José Crossa • \\ Conxita Royo
}

Received: 18 July 2013 / Accepted: 3 March 2014/Published online: 22 March 2014

(C) The Author(s) 2014. This article is published with open access at Springerlink.com

\begin{abstract}
With the aim of identifying durum wheat landraces (LR) with a potential use in breeding programs for gluten strength enhancement, the allelic combinations present at five glutenin loci were determined in a collection of 155 LR from 21 Mediterranean countries. A set of 18 modern cultivars (MC) was used for comparison. Gluten strength was determined by SDS-sedimentation test on grain samples from field experiments conducted during 3 years. A total number of 131 different allelic/banding pattern combinations were found. Taking together high (HMW-) and low (LMW-) molecular weight glutenin subunit loci resulted in 126 combinations in LR, but only nine in $\mathrm{MC}$, which are characterized for having strong gluten. Two LMW-2 type models were identified in the collection and LMW-1 types were absent. LMW-2 was present in $78 \%$ of MC, including the only three with outstanding gluten strength (Ocotillo, Claudio and Meridiano), while $14 \%$ of the LR had LMW-2 and $6 \%$ LMW-2- ${ }^{-}$. In the LR a known combination LMW-2 (aaa) and three new ones had a
\end{abstract}

R. Nazco · D. Villegas · C. Royo $(\bowtie)$

Field Crops Program, Institute for Food and Agricultural Research and Technology (IRTA), Avda. Rovira Roure 191, 25198 Lleida, Spain

e-mail: conxita.royo@irta.es

\section{R. J. Peña · K. Ammar · J. Crossa}

International Maize and Wheat Improvement Center (CIMMYT), Km 45 carretera Mex-Veracruz, El Batan,

Texcoco 56130, Mexico positive effect on the gluten strength. LMW-2 models were found in high frequency in LR from Italy and the three Maghreb countries; from medium to low frequencies in genotypes from Turkey, Jordan, Lebanon, Portugal and Spain, and were absent in the remaining countries. The large variability found in LR proved their potential value in breeding to broaden the genetic basis of gluten quality improvement. Genotypes interesting for breeding purposes are identified.

Keywords Gluten quality · Glutenin subunits ·

LMW models · Mediterranean landraces .

Triticum durum
Abbreviations
SDS Sodium dodecyl sulfate
HMW-GS High molecular weight glutenin subunit
LMW-GS Low molecular weight glutenin subunit
GS Glutenin subunit
LR Landraces
MC Modern cultivar

\section{Introduction}

Durum wheat (Triticum durum) is one of the oldest cultivated cereal species in the world. The earliest wheats, dated to approximately 10,000 years BP, were domesticated in the Fertile Crescent, a region extending from the coast of Israel to South-eastern Turkey and westwards through Syria, Iraq and western Iran 
(Feldman 2001). Durum wheat spread from this region westward into the Mediterranean basin, reaching the Iberian Peninsula around 7,000 years BP (Feldman 2001; MacKey 2005). It is widely accepted that durum wheat entered to North Africa and the Iberian Peninsula from the South of Italy (MacKey 2005). However, recent findings based on the genetic similarities between landraces (LR) from the Maghreb countries and those from Spain and Portugal have suggested North Africa as an additional route for wheat introduction in the Iberian Peninsula (Moragues et al. 2006c, 2007).

During the process of migration from the east to the west of the Mediterranean Basin durum wheat underwent a gradual adaptation to a large number of different specific environments (Moragues et al. 2006a, b). Natural and human selection resulted in a wide diversity of local LR specifically adapted to different agro-ecological areas. These dynamic populations, with distinct identity, are considered to be genetically more diverse than the currently cultivated varieties, locally adapted and associated with traditional farming systems (Camacho Villa et al. 2005). With the exception of Italy, where wheat breeding started at the beginning of the twentieth century, LR were mostly grown around the Mediterranean Basin until the advent of the Green Revolution in the late 1960s. The gradual replacement of traditional LR by improved, more homogeneous and productive semidwarf cultivars obtained from breeding programs, resulted in a loss of genetic diversity or genetic erosion starting from the late 60s in Southern Europe and during the 1970-80s in Northern Africa. Nowadays LR are considered a natural reservoir of the genetic variation within the species and one of the most important sources for potentially favorable genes/ alleles to be used in breeding programs.

Mediterranean countries represent the most important durum producing and importing region and the largest consumer of durum wheat products (Royo et al. 2009). Durum wheat is usually grown under rainfed conditions, in environments with large climate fluctuations in which terminal drought and heat are the most frequent stresses constraining grain yield. However, in most cases, environmental conditions during grain filling allow for the production of grain with the high quality standards demanded by the industry, provided requirements of functional attributes, such as gluten strength, are met.
The pasta cooking quality of durum wheat is largely affected by gluten strength, which is commonly and conveniently evaluated using the SDS-sedimentation test. Gluten strength depends on the composition of gliadins and glutenins, proteins stored in the grain endosperm, with glutenins being the most influential. The glutenin subunits (GS), can be identified according to their mobility in sodium dodecyl sulphate polyacrilamide gel electrophoresis (SDS-PAGE); they can be separated into high (HMW-GS) and low (LMW-GS) molecular weight glutenin subunits. The electrophoretic profile of these proteins has been used as a tool for identification of cultivars, pedigree analysis and population characterization because they offer a measure of genetic diversity within and between populations (Nevo and Payne 1987). The HMW-GS are encoded by a gene complex at the Glu- 1 loci (Glu-Al and Glu-Bl), located on the long arm of group-1 homologous chromosomes (Singh and Shepherd 1988; Shewry et al. 1992). The LMW-GS are encoded by a gene complex at the Glu-3 loci (Glu-A3 and $G l u-B 3$ ), and at the Glu-B2 locus (Ruiz and Carrillo 1993; Liu 1995), mapping to the short arms of group-1 homologous chromosomes.

Studies of the relationship between Glu-1 allelic composition and gluten quality have produced contrasting results in durum wheat. While some authors reported a positive association between certain $G l u-B 1$ allelic variants and gluten strength (Boggini and Pogna 1989), others found weak relationships (DuCros 1987). However, a positive association has been found between HMW-GS composition, particularly among LR, and the bread making quality of both bread and durum wheat (Tarekegne and Labuschagne 2005).

Early studies identified two allelic combinations for LMW-GS patterns in durum wheat, named LMW-1 model and LMW-2 model. These models are associated with $\gamma-42$ and $\gamma-45$ gliadin, respectively, although relationships with high gluten strength are likely to be caused by LMW glutenin subunits linked to $\gamma-45$ gliadin (Payne et al. 1984). Subsequent work found additional models or patterns, such as LMW-1 $1^{-}$, LMW-2 ${ }^{-}$and LMW-2* (Carrillo et al. 1990). LMW-2 and $\mathrm{LMW}-2^{-}$have been related to high gluten strength, while LMW-1 and LMW-1 ${ }^{-}$to poor quality (Carrillo et al. 1990; Pogna et al. 1990). Several authors have demonstrated that the strength of durum wheat gluten depends mostly on the allelic variation in LMW-GS (Ruiz and Carrillo 1995; Vázquez et al. 1996). 
Table 1 Country of origin of the 155 landraces included in the study and number of HMW-GS and LMW-GS allelic/banding pattern combinations detected by SDS-PAGE

\begin{tabular}{|c|c|c|}
\hline Country of origin & \multicolumn{2}{|c|}{ Number of entries } \\
\hline Algeria & \multicolumn{2}{|c|}{6} \\
\hline Bulgaria & \multicolumn{2}{|c|}{2} \\
\hline Croatia & \multicolumn{2}{|c|}{5} \\
\hline Cyprus & \multicolumn{2}{|c|}{3} \\
\hline Egypt & \multicolumn{2}{|c|}{11} \\
\hline France & \multicolumn{2}{|c|}{6} \\
\hline Greece & \multicolumn{2}{|c|}{3} \\
\hline Israel & \multicolumn{2}{|c|}{6} \\
\hline Italy & \multicolumn{2}{|c|}{13} \\
\hline Jordan & \multicolumn{2}{|c|}{8} \\
\hline Lebanon & \multicolumn{2}{|c|}{7} \\
\hline Libya & \multicolumn{2}{|c|}{1} \\
\hline Macedonia & \multicolumn{2}{|c|}{6} \\
\hline Montenegro & \multicolumn{2}{|c|}{5} \\
\hline Morocco & \multicolumn{2}{|c|}{11} \\
\hline Portugal & \multicolumn{2}{|c|}{11} \\
\hline Serbia & \multicolumn{2}{|c|}{3} \\
\hline Spain & \multicolumn{2}{|c|}{28} \\
\hline Syria & \multicolumn{2}{|c|}{4} \\
\hline Tunisia & \multicolumn{2}{|c|}{5} \\
\hline Turkey & \multicolumn{2}{|c|}{11} \\
\hline Combination & Landraces & Modern \\
\hline HMW glutenin & 32 & 4 \\
\hline LMW glutenin & 98 & 5 \\
\hline LMW model & 2 & 1 \\
\hline HMW and LMW glutenin & 126 & 9 \\
\hline
\end{tabular}

Allelic variation at the Glu-B3 locus seems to affect much more gluten quality than the allelic differences at Glu-A3 (Vázquez et al. 1996). Nieto-Taladriz et al. (1997) proposed a nomenclature for the commonly used LMW-models taking into account the specific LMW-GS encoded at the Glu-A3, Glu-B3 and Glu-B2 loci.

The present study was conducted with a set of 155 durum wheat LR from 21 Mediterranean countries and 18 representative modern cultivars (MC) to: (1) characterize and evaluate the frequency in the collection of the individual combinations of alleles/banding patterns of both HMW-GS and LMW-GS considered together, (2) identify those combinations showing significant effect on gluten strength, consequently in pasta cooking quality, and the LR carrying glutenin combinations with a potential use in breeding programs, and (3) assess whether a geographic distribution exists in the population according to the LMWmodels detected.

\section{Materials and methods}

Experimental setup

Plant material consisted of a collection of 155 durum wheat LR and old varieties derived from them, from 21 Mediterranean countries including the major durum producers/users (Table 1) and 18 representative MC. LR were selected-from a larger collection of 231 accessions-on the basis of their genetic variability determined by 33 SSR-markers (Nazco et al. 2012). Seeds provided by public gene banks (Centro de Recursos Fitogenéticos INIA-Spain, ICARDA Germplasm Bank, and USDA Germplasm Bank) were increased in bulk and purified by eliminating off types. The modern set included seven Spanish, five CIMMYT-derived, four Italian, and one French cultivar, as well as the US desert durum cultivar Ocotillo. Increase plots were planted in the same field in years previous to each experiment to ensure a common origin for seeds of all lines.

The collection was grown during 2007, 2008 and 2009 crop seasons in Gimenells $\left(41^{\circ} 40^{\prime} \mathrm{N}, 0^{\circ} 20^{\prime} \mathrm{E}\right.$, and $200 \mathrm{~m}$ a.s.1.) in Lleida province (North-eastern Spain). Experiments consisted on non-replicated plots of $6 \mathrm{~m}^{2}$ (comprising eight $5-\mathrm{m}$ rows, spaced $0.15 \mathrm{~m}$ apart), arranged in a modified augmented row-column design with three replicated checks (cultivars 'Claudio', 'Simeto' and 'Vitron'). Sowing density was adjusted to 250 viable seeds $\mathrm{m}^{-2}$. Water input was 208,308 and $237 \mathrm{~mm}$ in 2007, 2008 and 2009, respectively. Weeds and diseases were controlled according to standard cultural practices. Plots were mechanically harvested at commercial maturity. Experimental details may be found in Nazco et al. (2012).

\section{Gluten strength}

A sample of about $250 \mathrm{~g}$ of harvest-mature grain was randomly drawn from each plot, cleaned and $1 \mathrm{~g}$ of whole grain flour sample was used to determine gluten strength by the sodium dodecyl sulfate (SDS)- 
sedimentation test, following the method of Axford et al. (1978), further modified by Peña et al. (1990), using $25 \mathrm{ml}$ graduate cylinders.

Glutenin composition

Electrophoretic analysis (one dimensional SDSPAGE) of high and low molecular weight glutenin subunit composition at 5 loci (Glu-Al, Glu-Bl, Glu$A 3, G l u-B 3$, and $G l u-B 2$ ) was performed according to Peña et al. (2004), and subunits scored following the nomenclature of Nieto-Taladriz et al. (1997) and Martinez et al. (2004). The banding patterns/alleles with a frequency below 0.05 were classified as rare.

\section{Statistical analysis}

Raw data were fitted to a linear mixed model with the check cultivars as fixed effects, and the plot row/ column number or coordinates within the experiment as well as genotype as random effects. Restricted maximum likelihood (REML) was used to estimate the variance components and to produce the best linear unbiased predictors (BLUPs) for the gluten strength data of each cultivar in each year (MIXED procedure of the SAS-STAT statistical package (SAS Institute Inc. 2009).

Accessions were assigned to the following groups according to their mean SDS-sedimentation value across experiments: outstanding ( $\mathrm{SDS} \geq 11$ ), very high $(10<$ SDS $<11)$, high $(9 \leq \operatorname{SDS} \leq 10)$, medium $(7 \leq$ SDS $<9)$ and low (SDS $<7)$. A standard ANOVA, in which the genotype effect was partitioned according to this classification, was conducted with the BLUPs of gluten strength data. Means were compared by the Student-Newman-Keuls (SNK) test at $P=0.05$.

The effect of each combination of glutenin subunits on gluten strength was calculated as the difference between the mean SDS-sedimentation test values of the accessions carrying it and that of the remainder accessions. The FREQ procedure of the SAS-STAT statistical package was used to conduct a Fisher's exact test on each significant allelic/banding pattern combination, to determine whether there were any significant differences between the gluten-strength groups with regard to each combination frequency. The frequency of the combination of LMW models by country was used to perform hierarchical cluster analysis by the Ward method of the JMP V.8 software (SAS Institute Inc. 2009). Genetic diversity was calculated with the D index (Weir 1996), according to the following expression:

$\mathrm{D}_{\mathrm{j}}=1-\Sigma \mathrm{p}_{\mathrm{ij}}^{2}$

where $\mathrm{p}$ is the frequency of the ith allelic/banding pattern combination at jth country.

\section{Results}

A total number of 114 individual banding patterns, potentially $G l u-1 / G l u-3 / G l u-2$ allele-specific, were identified in the collection, with the following distribution: 5 and 20 HMW-GS encoded by the Glu-Al and Glu-B1 loci respectively, and 15, 72 and 2 LMW-GS encoded by the Glu-A3, Glu-B3 and Glu-B2 loci, respectively (Nazco et al. 2013). Considering jointly both glutenin groups (HMW-GS and LMW-GS), a total of 131 combinations were detected in the whole collection, with 126 identified in LR and 9 in MC. Detailed list of these combinations are presented in Table 2. In LR, 32 combinations were detected at the Glu-1 loci and 98 at the Glu-2 and Glu-3 loci. In MC, the number of combinations was much more reduced, with four and five detected at the Glu- 1 and Glu-2/Glu3 loci, respectively (Table 1 ). The large number of combinations identified in the LR resulted in a very low frequency of many of them. The most frequent combination in MC was combination1 (null allele at Glu-Al and subunit $7+8$ at $G l u-B 1$ for HMW-GS loci, and bands $6,2+4+15+19$ and 12 at Glu-A3, Glu-B3 and Glu-B2 loci, respectively), which appeared in $7(38.89 \%)$ entries (Table 2). On the other hand, combination 21, whose only difference with combination 1 was the banding pattern at Glu-B1 locus (HMW-GS 20 instead of HMW-GS $7+8$ ), was the most common in the LR and was recorded in 13 $(8.39 \%)$ of them. All the allelic/banding pattern combinations identified in MC were also present in the LR (Table 2).

No LMW-1 type models were found in the present collection. Two LMW models, LMW-2 and LMW-2 ${ }^{-}$, were identified, with LMW-2 represented by 2 different combinations (Table 2). Model LMW-2 was the most frequent, both in LR $(14.19 \%)$ and in MC (77.78\%). LMW-2 (aaa) sub-model was part of the 
Table 2 Allelic/banding pattern combinations and LMW models identified and their frequency calculated for 155 landraces and 18 representative modern cultivars (MC)

\begin{tabular}{|c|c|c|c|c|c|c|c|}
\hline \multirow{2}{*}{$\begin{array}{l}\text { Combination } \\
\text { number }\end{array}$} & \multicolumn{2}{|c|}{ High molecular weight } & \multicolumn{3}{|c|}{ Low molecular weight } & \multicolumn{2}{|c|}{ Frequency $(\%)$} \\
\hline & Glu-Al & Glu-B1 & Glu-A3 & Glu-B3 & Glu-B2 & Landraces & Modern \\
\hline 1 & Null & $7+8$ & 6 & $2+4+15+19$ & 12 & 0.65 & 38.89 \\
\hline 2 & Null & $7+8$ & 6 & $2+4+15+18$ & 12 & 0.65 & - \\
\hline 3 & Null & $7+8$ & 6 & $4+15+19$ & 12 & - & 5.56 \\
\hline 4 & Null & $7+8$ & 6 & $4+16+19$ & 12 & 0.65 & - \\
\hline 5 & Null & $7+8$ & 6 & $2+4+14+15+16$ & Null & 0.65 & - \\
\hline 6 & Null & $7+8$ & Null & $1+3+13+16$ & Null & 0.65 & - \\
\hline 7 & Null & $7+8$ & Null & $2+4+15+18$ & Null & 0.65 & - \\
\hline 8 & Null & $7+8$ & 11 & $3+14+16$ & Null & 0.65 & - \\
\hline 9 & Null & $7+8$ & 11 & $2+4+14+15+16$ & Null & 0.65 & - \\
\hline 10 & Null & $7+8$ & 5 & $1+15+17$ & Null & 0.65 & - \\
\hline 11 & Null & $7+8$ & 5 & $3+7+16+19$ & Null & 0.65 & - \\
\hline 12 & Null & $7+8$ & 5 & $9+13+17+18$ & 12 & 0.65 & - \\
\hline 13 & Null & $7+8$ & $5+11$ & $14+16$ & 12 & 0.65 & - \\
\hline 14 & Null & $7+8$ & $5+11$ & $14+17$ & Null & 0.65 & - \\
\hline 15 & Null & $7+8$ & $6+11$ & $2+4+15+19$ & Null & - & 11.11 \\
\hline 16 & Null & $7+8$ & $6+20$ & $2+4+15+19$ & Null & 1.29 & - \\
\hline 17 & Null & $7+8$ & $11+10$ & $7+13+19$ & Null & 0.65 & - \\
\hline 18 & Null & 20 & 6 & $2+4+13+17$ & Null & 0.65 & - \\
\hline 19 & Null & 20 & 6 & $2+4+13+16+17$ & Null & 0.65 & - \\
\hline 20 & Null & 20 & 6 & $2+4+15+16$ & 12 & 0.65 & - \\
\hline 21 & Null & 20 & 6 & $2+4+15+19$ & 12 & 8.39 & 11.11 \\
\hline 22 & Null & 20 & 6 & $2+4+16+17$ & 12 & 0.65 & - \\
\hline 23 & Null & 20 & 6 & $2+4+16+19$ & 12 & 0.65 & - \\
\hline 24 & Null & 20 & 6 & $2+4+17$ & 12 & 0.65 & - \\
\hline 25 & Null & 20 & Null & $2+4+15+16$ & 12 & 0.65 & - \\
\hline 26 & Null & 20 & Null & $2+4+15+19$ & 12 & 4.52 & - \\
\hline 27 & Null & 20 & Null & $2+4+16+19$ & Null & 0.65 & - \\
\hline 28 & Null & 20 & 11 & $2+4+14+18$ & Null & 0.65 & - \\
\hline 29 & Null & 20 & 11 & $2+4+15+19$ & Null & 0.65 & - \\
\hline 30 & Null & 20 & 11 & $9+13+17$ & 12 & 0.65 & - \\
\hline 31 & Null & 20 & 5 & $3+7+14+15+19$ & Null & 0.65 & - \\
\hline 32 & Null & 20 & $5+11$ & $14+17$ & 12 & 0.65 & - \\
\hline 33 & Null & 20 & $5+11$ & $3+15+19$ & Null & - & 5.56 \\
\hline 34 & Null & 20 & $6+11$ & $2+4+14+18$ & Null & 0.65 & - \\
\hline 35 & Null & 20 & $6+11$ & $4+15+19$ & Null & - & 5.56 \\
\hline 36 & Null & 20 & $6+11$ & $2+4+15+19$ & Null & 0.65 & - \\
\hline 37 & Null & 20 & 10 & $14+18$ & Null & 0.65 & - \\
\hline 38 & Null & $6+8$ & 6 & $2+4+15+19$ & 12 & 2.58 & 11.11 \\
\hline 39 & Null & $6+8$ & 6 & $2+4+15+18$ & 12 & 1.29 & - \\
\hline 40 & Null & $6+8$ & 6 & $2+4+15+18$ & Null & 0.65 & - \\
\hline 41 & Null & $6+8$ & 6 & $4+15+19$ & Null & 0.65 & - \\
\hline 42 & Null & $6+8$ & Null & $2+4+15+19$ & 12 & 1.29 & - \\
\hline 43 & Null & $6+8$ & Null & $2+4+15+19$ & Null & 0.65 & - \\
\hline
\end{tabular}


Table 2 continued

\begin{tabular}{|c|c|c|c|c|c|c|c|}
\hline \multirow{2}{*}{$\begin{array}{l}\text { Combination } \\
\text { number }\end{array}$} & \multicolumn{2}{|c|}{ High molecular weight } & \multicolumn{3}{|c|}{ Low molecular weight } & \multicolumn{2}{|c|}{ Frequency $(\%)$} \\
\hline & Glu-Al & Glu-B1 & Glu-A3 & Glu-B3 & Glu-B2 & Landraces & Modern \\
\hline 44 & Null & $6+8$ & Null & $1+3+14+18$ & Null & 0.65 & - \\
\hline 45 & Null & $6+8$ & 11 & $2+4+15+18+19$ & Null & 0.65 & - \\
\hline 46 & Null & $6+8$ & 11 & $3+14+16$ & Null & 0.65 & - \\
\hline 47 & Null & $6+8$ & 5 & $14+17$ & 12 & 0.65 & - \\
\hline 48 & Null & $6+8$ & 5 & $3+9+14+17+18$ & Null & 0.65 & - \\
\hline 49 & Null & $6+8$ & $5+10+11$ & $3+14+16+18$ & Null & 0.65 & - \\
\hline 50 & Null & $6+8$ & $5+11$ & $13+16$ & Null & 0.65 & - \\
\hline 51 & Null & $6+8$ & $5+11$ & $3+15+18+19$ & Null & 0.65 & - \\
\hline 52 & Null & $6+8$ & $5+11$ & $14+16+19$ & Null & 0.65 & - \\
\hline 53 & Null & $6+8$ & $6+10$ & $2+4+13+16+19$ & Null & 0.65 & - \\
\hline 54 & Null & $6+8$ & $6+11$ & $2+4+15+19$ & Null & 0.65 & 5.56 \\
\hline 55 & Null & $6+8$ & $6+11$ & $2+4+13+15+18$ & Null & 0.65 & - \\
\hline 56 & Null & $6+8$ & $6+20$ & $2+4+14+15+19$ & Null & 0.65 & - \\
\hline 57 & Null & $6+8$ & 10 & $2+4+14+17+18$ & Null & 0.65 & - \\
\hline 58 & Null & $6+8$ & $10+11$ & $14+17+18$ & Null & 0.65 & - \\
\hline 59 & Null & $6+8$ & $11+20$ & $14+15+18+19$ & Null & 0.65 & - \\
\hline 60 & Null & $6++17$ & $5+11$ & $14+17$ & Null & 0.65 & - \\
\hline 61 & Null & $6+18$ & $5+11$ & $14+17$ & Null & 0.65 & - \\
\hline 62 & Null & $6+18$ & 6 & $2+4+15+16$ & 12 & 0.65 & - \\
\hline 63 & Null & $6+18$ & 11 & $3+13+15+19$ & Null & 0.65 & - \\
\hline 64 & Null & $6+22$ & 6 & $2+4+15+19$ & Null & 0.65 & - \\
\hline 65 & Null & $7+8-$ & $5+11$ & $14+16$ & 12 & 0.65 & - \\
\hline 66 & Null & $7+17$ & $6+11$ & $4+15+19$ & Null & - & 5.56 \\
\hline 67 & Null & $7+22$ & 6 & $2+4+16+17$ & Null & 0.65 & - \\
\hline 68 & Null & $13+16$ & 6 & $3+15+19$ & Null & 0.65 & - \\
\hline 69 & Null & $13+16$ & 11 & $1+3+14+18$ & Null & 0.65 & - \\
\hline 70 & Null & $13+16$ & Null & $2+4+15+18$ & Null & 0.65 & - \\
\hline 71 & Null & $13+16$ & Null & $2+4+15+19$ & Null & 0.65 & - \\
\hline 72 & Null & $14++18$ & Null & $2+4+16+17$ & 12 & 0.65 & - \\
\hline 73 & Null & $14+15$ & 6 & $2+4+15+19$ & 12 & 0.65 & - \\
\hline 74 & Null & $14+15$ & 11 & $2+4+14+18+19$ & Null & 0.65 & - \\
\hline 75 & Null & $19+22$ & 10 & $3+8+14+17$ & 12 & 0.65 & - \\
\hline 76 & Null & $19+22$ & $6+11$ & $16+18$ & Null & 0.65 & - \\
\hline 77 & Null & $20+18$ & $6+11$ & $2+4+7+13+17+19$ & Null & 0.65 & - \\
\hline 78 & 1 & $7+8$ & 6 & $2+4+15+18$ & 12 & 0.65 & - \\
\hline 79 & 1 & $7+8$ & $5+11$ & $3+9+15+18$ & Null & 0.65 & - \\
\hline 80 & 1 & $7+8$ & $10+11$ & $3+14+17+19$ & Null & 0.65 & - \\
\hline 81 & 1 & $7+8$ & $10+11$ & $15+18$ & Null & 0.65 & - \\
\hline 82 & 1 & 20 & 6 & $2+4+15+18$ & 12 & 0.65 & - \\
\hline 83 & 1 & 20 & 6 & $2+4+15+19$ & 12 & 0.65 & - \\
\hline 84 & 1 & 20 & 6 & $2+4+15+19$ & Null & 0.65 & - \\
\hline 85 & 1 & 20 & 6 & $2+4+14+15+19$ & Null & 0.65 & - \\
\hline 86 & 1 & 20 & Null & $3+15+17$ & 12 & 0.65 & - \\
\hline
\end{tabular}


Table 2 continued

\begin{tabular}{|c|c|c|c|c|c|c|c|}
\hline \multirow{2}{*}{$\begin{array}{l}\text { Combination } \\
\text { number }\end{array}$} & \multicolumn{2}{|c|}{ High molecular weight } & \multicolumn{3}{|c|}{ Low molecular weight } & \multicolumn{2}{|c|}{ Frequency $(\%)$} \\
\hline & Glu-A1 & Glu-B1 & Glu-A3 & Glu-B3 & Glu-B2 & Landraces & Modern \\
\hline 87 & 1 & 20 & Null & $2+4+15+18$ & Null & 0.65 & - \\
\hline 88 & 1 & 20 & Null & $2+4+15+19$ & 12 & 0.65 & - \\
\hline 89 & 1 & 20 & Null & $2+4+15+19$ & Null & 0.65 & - \\
\hline 90 & 1 & 20 & Null & $2+4+15+17+19$ & Null & 0.65 & - \\
\hline 91 & 1 & 20 & 11 & $2+4+16+17+18$ & Null & 0.65 & - \\
\hline 92 & 1 & 20 & $5+10$ & $9+15+17+19$ & Null & 0.65 & - \\
\hline 93 & 1 & 20 & $5+10+11$ & $15+16+18$ & Null & 0.65 & - \\
\hline 94 & 1 & 20 & $5+11$ & $16+17+19$ & 12 & 0.65 & - \\
\hline 95 & 1 & 20 & $6+20$ & $2+4+15+19$ & 12 & 2.58 & - \\
\hline 96 & 1 & 20 & $10+11$ & $15+18$ & Null & 0.65 & - \\
\hline 97 & 1 & 20 & 20 & $2+4+15+19$ & 12 & 0.65 & - \\
\hline 98 & 1 & 20 & 20 & $2+4+15+19$ & 12 & 0.65 & - \\
\hline 99 & 1 & $6+8$ & Null & $2+4+15+19$ & Null & 0.65 & - \\
\hline 100 & 1 & $6+8$ & 11 & $2+4+13+17+18$ & Null & 0.65 & - \\
\hline 101 & 1 & $6+8$ & 5 & $1+15+17+18$ & 12 & 0.65 & - \\
\hline 102 & 1 & $6+8$ & $5+10+11$ & $16+19$ & Null & 1.29 & - \\
\hline 103 & 1 & 7 & Null & $2+4+15+19$ & Null & 0.65 & - \\
\hline 104 & 1 & $13+19$ & $6+11$ & $2+4+15+18+19$ & Null & 0.65 & - \\
\hline 105 & 1 & $13+18$ & 5 & $14+15+17$ & 12 & 0.65 & - \\
\hline 106 & 1 & 14 & $5+11$ & $1+14+17$ & Null & 0.65 & - \\
\hline 107 & 1 & $19+22$ & 10 & $15+16+17$ & 12 & 0.65 & - \\
\hline 108 & $1^{\prime}$ & $13+16$ & $5+11$ & $13+19$ & Null & 0.65 & - \\
\hline 109 & $1 / 2 * *$ & $7+17$ & $6+11$ & $2+4+15+18$ & Null & 0.65 & - \\
\hline 110 & $2 *$ & $7+8$ & $5+11$ & $9+15+18$ & Null & 0.65 & - \\
\hline 111 & $2 *$ & 20 & Null & $8+13+14+16$ & Null & 0.65 & - \\
\hline 112 & $2 *$ & $6+8$ & 10 & $2+4+14+17$ & Null & 0.65 & - \\
\hline 113 & $2 *$ & $6+8$ & 6 & $2+4+15+19$ & Null & 0.65 & - \\
\hline 114 & $2 *$ & $6+8$ & Null & $2+4+15+19$ & Null & 0.65 & - \\
\hline 115 & $2 *$ & $6+17$ & $5+10+11$ & $13+14+16$ & Null & 0.65 & - \\
\hline 116 & $2 *$ & $6+17$ & 5 & $9+15+17+18$ & Null & 0.65 & - \\
\hline 117 & $2 *$ & $6+18$ & $5+11$ & $14+17$ & 12 & 0.65 & - \\
\hline 118 & $2 *$ & 7 & 6 & $2+4+15+19$ & Null & 0.65 & - \\
\hline 119 & $2 *$ & 7 & 5 & $1+13+14+17$ & Null & 0.65 & - \\
\hline 120 & $2 *$ & 7 & 5 & $3+13+18$ & Null & 0.65 & - \\
\hline 121 & $2 *$ & 7 & $6+10$ & $2+4+17$ & 12 & 0.65 & - \\
\hline 122 & $2 *$ & 7 & Null & $2+4+15+18$ & Null & 0.65 & - \\
\hline 123 & $2 *$ & $7+17$ & 6 & $2+4+14+15+18$ & Null & 0.65 & - \\
\hline 124 & $2 *$ & $7+17$ & 5 & $1+3+14+15+18$ & Null & 0.65 & - \\
\hline 125 & $2 *$ & $7+17$ & 5 & $1+3+13+14+17$ & Null & 0.65 & - \\
\hline 126 & $2 *$ & $7+17$ & $6+11$ & $14+17$ & Null & 0.65 & - \\
\hline 127 & $2 *$ & $7+17$ & 11 & $14+17$ & Null & 0.65 & - \\
\hline 128 & $2 *$ & $7+17$ & Null & $1+7+15+16$ & Null & 0.65 & - \\
\hline 129 & $2 *$ & $14++18$ & Null & $2+4+9+13+17$ & Null & 0.65 & - \\
\hline
\end{tabular}


Table 2 continued

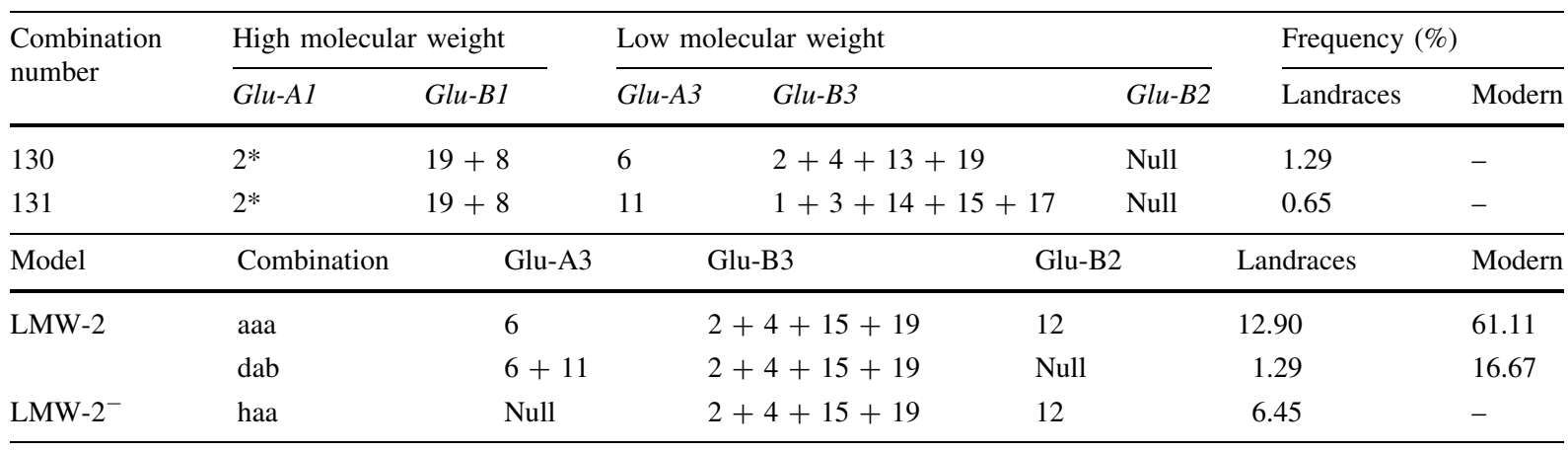

Previously reported combinations are in bold type

combinations identified as numbers 1, 21, 38, 73 and 83 in Table 2. It was the only model present in Algerian, Lebanese, Jordanian and Portuguese LRs, with frequencies of 83.3, 28.6, 12.5 and $9.1 \%$, respectively. LMW-2 (dab) sub-model was present in combinations numbers 15, 36 and 54 (Table 2), and exclusively found in two Spanish LR (Table 3). LMW-2 ${ }^{-}$corresponded to combinations 26,42 and 88 (Table 2) and was recorded in LR from Morocco (36.4\%), Italy (23.1\%), Tunisia (20.0\%), Turkey $(9.1 \%)$, and Spain $(3.6 \%)$. The only country having the three LMW sub-models combinations represented was Spain. Eleven, among the $18 \mathrm{MC}(61 \%)$, had LMW-2 (aaa) (Tables 2 and 3), while LMW-2 (dab) was detected in $17 \%$ of them, namely 'Amilcar','Svevo' and 'Vitronero'.

The overall genetic diversity index (D), calculated for the allelic/banding pattern combinations, was higher for the LR than for MC (Table 4). Mean D values by country ranged from 0.50 to 0.96 with the highest for LR from Spain, Portugal, Egypt, Jordan and Lebanon, and the lowest for LR from Bulgaria, Algeria, Serbia, Greece and Cyprus (Table 4).

The results of the ANOVA for gluten strength (data not shown) indicated that both year and genotype effects were significant $(P<0.0001)$, explaining respectively 9 and $73 \%$ of the total variation. The partitioning of the genotype effect into its components allowed quantifying the percentage of the genotypic variance accounted by differences between the five groups of gluten strength, and differences within each of them. The results revealed that differences between groups accounted for $91.5 \%$ of the genotypic effect, while variability within each sedimentation group was not significant.
The effect of combinations and models/banding patterns on gluten strength, the difference between the SDS-value of the accessions carrying a given combination, and those which do not, was calculated for LR and MC separately (Table 5). Whereas no combinations or LMW models had a significant effect on the gluten strength of MC, two individual combinations, as well as two LMW models significantly influenced the gluten strength of LR. The 13 LR $(8.39 \%)$ exhibiting combination 21 (Table 2), had an average SDS-value $1.30 \mathrm{ml}$ higher than those carrying alternative combinations. Similarly, the SDS-value of the 7 LR carrying combination 26 , absent in MC, and only differing from combination 21 by the presence of the Glu-A3 null allele (Table 2), was $1.19 \mathrm{ml}$ greater than that of the remainder LR (Table 5). Variant aaa of the LMW-2 model (bands 6, $2+4+15+19$ and 12 at Glu-A3, Glu-B3 and Glu-B2 loci, respectively), included in combination 21, and model LMW-2included in combination 26 , significantly increased the gluten strength of LR (Table 5). Only three among the rare combinations found in the LR had a significant effect on gluten strength, the three of them, drastically increased it. Combination 95, which, on average, increased the SDS-value by $2.33 \mathrm{ml}$, was found in four Turkish LR (Table 3). Combinations 45 and 123, associated with very high SDS-sedimentation values, were detected in the French cultivar 'Trigo Glutinoso' and in an Egyptian accession (PI-366109), respectively (Table 3 ).

The frequency of allelic/banding pattern combinations and models with significant effect on gluten strength was calculated for each of the five gluten strength groups considered in the ANOVA, distinguishing between already known combinations and 
Table 3 Landraces and MC in which the allelic/banding pattern combinations were identified

\begin{tabular}{|c|c|c|c|c|}
\hline $\begin{array}{l}\text { Combination } \\
\text { number }\end{array}$ & Landraces & Modern & $\begin{array}{l}\text { Combination } \\
\text { number }\end{array}$ & Landraces \\
\hline 1 & JO: Salti na Zinia & $\begin{array}{l}\text { Claudio, Gallareta, } \\
\text { Jupare, Meridiano, } \\
\text { Simeto, Sula, Vitron }\end{array}$ & 67 & IS: Etith \\
\hline 2 & TUR: BGE-019263 & & 68 & SP: Arisnegro de Tenerife \\
\hline 3 & & Ancalei & 69 & GR: IG-96851 \\
\hline 4 & EG: PI-60726 & & 70 & AL: IG-92967 \\
\hline 5 & SY: IG-95841 & & 71 & SP: Farto canifino \\
\hline 6 & SY: IG-95931 & & 72 & SP: Pinet \\
\hline 7 & IT: Cicirelo & & 73 & AL: IG-92895 \\
\hline 8 & FR: Tounse & & 74 & CY: Muri \\
\hline 9 & EG: Sinai No.8 & & 75 & PO: Espanhol \\
\hline 10 & IS: Juljulith & & 76 & MO: Cobros \\
\hline 11 & LE: PI-182666 & & 77 & $\begin{array}{l}\text { MO: Maghoussa } \\
\text { Amizmiz }\end{array}$ \\
\hline 12 & SP: Candeal de Salamanca & & 78 & CY: IG-82549 \\
\hline 13 & IT: Aziziah 17/45 & & 79 & SP: Blanco de Corella \\
\hline 14 & LI: Tripshiro & & 80 & MA: PI-405908 \\
\hline 15 & & Amilcar, Svevo & 81 & GR: Rapsani \\
\hline 16 & IS: PI-572901, PI-572903 & & 82 & CY: Vroulos \\
\hline 17 & IS: Abu Fashit & & 83 & TUR: Mindium \\
\hline 18 & JO: Safra Maan & & 84 & $\begin{array}{l}\text { SP: Raspinegro de Alcalá } \\
\text { Guadaira }\end{array}$ \\
\hline 19 & JO: Zoghbiyeh Safra & & 85 & EG: Reading \\
\hline 20 & LE: PI-182667 & & 86 & SE: Belgrade 9 \\
\hline 21 & $\begin{array}{l}\text { AL: Dur de Medeah, IG-93030, IG-93621 } \\
\text { IT: Capeiti 8, Hymera, Razza 96, Senatore } \\
\text { Capelli } \\
\text { LE: IG-84856; MO: Mahmoudi C, Morocco } \\
\text { TU: Biskri, Realforte; TUR: BGE018351 }\end{array}$ & Bolo, Hispasano & 87 & IT: Balilla Falso \\
\hline 22 & LE: Hourah & & 88 & TUR: BGE019266 \\
\hline 23 & MO: Haj Mouline & & 89 & PO: Tremes rijo \\
\hline 24 & JO: PI-420946 & & 90 & MA: PI-345249 \\
\hline 25 & JO: Safra Jerash & & 91 & SE: PI-378303 \\
\hline 26 & $\begin{array}{l}\text { IT: Carlo jucci, Razza 208, Trinakria MO: } \\
\text { Merzaga, Oned Zenati, Red Beard SP: } \\
\text { Recio de Canete }\end{array}$ & & 92 & MA: PI-362629 \\
\hline 27 & LE: PI-182671 & & 93 & MA: PI-374658 \\
\hline 28 & SP: Claro de Balazote & & 94 & MA: PI-345260 \\
\hline 29 & TUR: BGE-018354 & & 95 & $\begin{array}{l}\text { TUR: BGE018353, } \\
\text { BGE019262, } \\
\text { BGE019264, } \\
\text { BGE019265 }\end{array}$ \\
\hline 30 & SE: PI-585195 & & 96 & GR: Mavraani \\
\hline 31 & MO: Maghoussa & & 97 & TUR: BGE-018192 \\
\hline 32 & JO: Zugbieh Sutra & & 98 & TUR: BGE-019270 \\
\hline 33 & & Arment & 99 & PO: Dezassete \\
\hline
\end{tabular}


Table 3 continued

\begin{tabular}{|c|c|c|c|c|}
\hline $\begin{array}{l}\text { Combination } \\
\text { number }\end{array}$ & Landraces & Modern & $\begin{array}{l}\text { Combination } \\
\text { number }\end{array}$ & Landraces \\
\hline 34 & SP: Blancal & & 100 & CR: PI-435057 \\
\hline 35 & & Astigi & 101 & CR: PI-345441 \\
\hline 36 & SP: Recio de Almería & & 102 & $\begin{array}{l}\text { SP: Griego de Baleares, } \\
\text { Gros de Cerdaña }\end{array}$ \\
\hline 37 & BU: Lozen 76 & & 103 & MON: PI-435024 \\
\hline 38 & $\begin{array}{l}\text { AL: IG-94009; LE: Reyati } \\
\text { PO: Raspinegro; SP: Farto }\end{array}$ & Ocotillo, Senadur & 104 & $\begin{array}{l}\text { PO: Lobeiro de grao } \\
\text { escuro }\end{array}$ \\
\hline 39 & IT: Carlantino; SP: Enano de Andujar & & 105 & MO: Zoco Yebel Hebil \\
\hline 40 & SP: Ruso & & 106 & SP: Rubio de Montijo \\
\hline 41 & TU: Hamira & & 107 & CR: PI-345442 \\
\hline 42 & MO: Ble Dur 250; TU: Louri AP 5 & & 108 & SP: Pisana canihueca \\
\hline 43 & TU: Souri & & 109 & EG: Giza 2 \\
\hline 44 & IT: IG-83905 & & 110 & PO: Alentejo \\
\hline 45 & FR: Trigo Glutinoso & & 111 & BU: Tchirpan \\
\hline 46 & FR: Lumillo & & 112 & SP: Verdial \\
\hline 47 & PO: Amarelo Barba Preta & & 113 & SP: Basto Duro \\
\hline 48 & SP: Colorado de Jerez & & 114 & CR: Dalmatia 1 \\
\hline 49 & SP: Azulejo de Villa del Río & & 115 & SY: IG-95812 \\
\hline 50 & EG: PI-60727 & & 116 & MA: PI-362638 \\
\hline 51 & PO: Raposinho & & 117 & JO: Horani Howawi \\
\hline 52 & FR: De Santa Marta & & 118 & CR: Dalmatia 3 \\
\hline 53 & SP: Raspinegro Canario & & 119 & SP: Blanquillón de Boñar \\
\hline 54 & SP: Rubio de Miajadas & Vitronero & 120 & MON: PI-435043 \\
\hline 55 & SP: Alonso & & 121 & SP: Heraldo del Rhin \\
\hline 56 & PO: Marques & & 122 & MON: PI-345357 \\
\hline 57 & SP: Entrelargo de Montijo & & 123 & EG: PI-366109 \\
\hline 58 & FR: Rubio enlargado d'Atlemteje & & 124 & EG: PI-576803 \\
\hline 59 & FR: Beladi Rouge & & 125 & EG: Girgeh \\
\hline 60 & IS: Hati & & 126 & EG: PI-113397 \\
\hline 61 & JO: Harani Auttma & & 127 & EG: PI-559973 \\
\hline 62 & SY: IG-95847 & & 128 & PO: Durazio Rijo Glabro \\
\hline 63 & LE: PI-182669 & & 129 & SP: Blanquillo \\
\hline 64 & PO: Anafil & & 130 & $\begin{array}{l}\text { MON: PI-435034, } \\
\text { PI-435038 }\end{array}$ \\
\hline 65 & IT: IG-83920 & & 131 & EG: Mishriki \\
\hline 66 & & Boabdil & & \\
\hline
\end{tabular}

See Table 2 for combinations description

Accessions codes: BGE-numbers are codes from the Centro de Recursos Fitogenéticos (I.N.I.A., Madrid), IG-numbers are codes from ICARDA Germplasm Bank. PI-numbers are codes from USDA Germplasm Bank

a Country codes: AL: Algeria, BU: Bulgaria, CR: Croatia, CY: Cyprus, EG: Egypt, FR: France, GR: Greece, IS: Israel, IT: Italy, JO: Jordan, LE: Lebanon, LI: Libya, MA: Macedonia, MON: Montenegro, MO: Morocco, PO: Portugal, SE: Serbia, SP: Spain, SY: Syria, TU: Tunisia, TUR: Turkey 
Table 4 Genetic diversity indices calculated for the allelic/ banding pattern combinations identified in 155 landraces (per country of origin) and 18 representative modern cultivars (MC)

\begin{tabular}{ll}
\hline & $\mathrm{D}$ \\
\hline Modern & 0.80 \\
Landraces & 0.98 \\
\hline Algeria & 0.67 \\
Bulgaria & 0.50 \\
Croatia & 0.80 \\
Cyprus & 0.67 \\
Egypt & 0.91 \\
France & 0.83 \\
Greece & 0.67 \\
Israel & 0.78 \\
Italy & 0.82 \\
Jordan & 0.88 \\
Lebanon & 0.86 \\
Libya & 0.00 \\
Macedonia & 0.83 \\
Montenegro & 0.72 \\
Morocco & 0.84 \\
Portugal & 0.91 \\
Serbia & 0.67 \\
Spain & 0.96 \\
Syria & 0.75 \\
Tunisia & 0.72 \\
Turkey & 0.81 \\
\hline &
\end{tabular}

those which have not yet been reported. All MC had SDS values $\geq 9 \mathrm{ml}$, corresponding to at least the high gluten strength class. Among them, 'Claudio', 'Meridiano' and 'Ocotillo' had the highest gluten strength and a common LMW-2 model (Table 6). The only difference between combinations 1 (present in 'Claudio' and 'Meridiano') and 38 (present in 'Ocotillo') was the banding pattern at $G l u-B 1$ locus, that is $7+8$ at combination 1 and $6+8$ at combination 38 (Table 2). Four among the MC with very high gluten strength had combinations previously described (Table 6), while new combinations were found in the cultivars 'Boabdil', 'Astigi' and 'Ancalei'. The pattern of 'Svevo' at LMW-2 model differed from that of 'Simeto', 'Sula' and 'Bolo' in the region corresponding to bands encoded by Glu-A3 and Glu-B2 loci. The variety 'Arment' was, among those with high gluten strength, the only one carrying a novel combination (number 33 in Table 2), while the most frequent in this group was combination one. 'Amilcar' and 'Vitronero' showed a different LMW-2 pattern than the other cultivars of this group (Table 6).

None of the combinations significantly increasing gluten strength were present in LR with SDS values lower than $7 \mathrm{ml}$ (Table 6). In addition, known combinations 21 and 26 were also missing in the five LR with outstanding SDS values. Combination 21 was detected in $2(14.3 \%)$ of the 14 LR with very high gluten strength, in 9 (29\%) of the 31 LR with high gluten strength and only in $2(2.8 \%)$ of the 71 accessions with medium gluten strength. One LR (7.1\%) of the 14 having very high SDS value, carried combination number 26 , which was also present in 4 $(12.9 \%)$ and $2(2.8 \%)$, respectively, of the LR with high and medium gluten strength. Unreported combinations 45, 95 and 123 were each identified in one (20\%) of the 5 LR with outstanding SDS-values. Combination 95 was also present in one $(7.1 \%)$ and 2 $(6.5 \%)$ of the LR with very high and high gluten strength, respectively (Table 6). LMW-2 and LMW$2^{-}$models were missing in LR with outstanding gluten strength. Model LMW-2 was the most frequent in LR with high gluten strength. Model LMW-2 ${ }^{-}$was present in $2(14.3 \%), 4(12.9 \%)$ and 4 (5.6\%) LR with very high, high and medium gluten strength, respectively and it was not detected in any LR with low gluten strength (Table 6). The results of the Fisher's exact test applied to the whole set of data revealed that for combinations 1, 21, 45, 95 and 123, model LMW-2 and sub-model aaa, the differences between gluten-strength groups were associated with the frequencies of the observed allelic/banding pattern combinations (Table 6). When the Fisher's text was conducted for LR and MC separately, no significant association appeared for MC, while for the LR the significant relationships coincided with the ones shown in Table 6 for the whole set of cultivars, except for combination number 1 , which became not statistically significant.

The genetic diversity indices calculated for each gluten strength group were greater for LR than for MC (bottom part of Table 6).

Clustering analysis based on the frequencies of the LMW models identified in the collection (Fig. 1) grouped countries of origins of LR in three branches corresponding to high frequencies (branch A), low frequencies (branch B) and absence of the LMW models (branch C). The highest frequencies for any 
Table 5 Known and not yet reported allelic/banding patterns combinations with significant effect on SDS-sedimentation volume $(\mathrm{ml})$ in the collection of 155 landraces and 18 representative modern cultivars (MC) and their frequencies

\begin{tabular}{|c|c|c|c|c|c|c|c|c|c|}
\hline & \multirow{2}{*}{$\begin{array}{l}\text { Combination } \\
\text { number }\end{array}$} & \multicolumn{4}{|l|}{ Landraces } & \multicolumn{4}{|l|}{ Modern } \\
\hline & & $\begin{array}{l}\text { Frequency } \\
(\%)\end{array}$ & $\begin{array}{l}\text { Present }^{\mathrm{a}} \\
(\mathrm{ml})(\mathrm{a})\end{array}$ & $\begin{array}{l}\text { Absent } \\
(\mathrm{ml})(\mathrm{b})\end{array}$ & $\begin{array}{l}\text { Main effect } \\
(\mathrm{ml})(\mathrm{a}-\mathrm{b})\end{array}$ & $\begin{array}{l}\text { Frequency } \\
(\%)\end{array}$ & $\begin{array}{l}\text { Present } \\
(\mathrm{ml})(\mathrm{a})\end{array}$ & $\begin{array}{l}\text { Absent } \\
(\mathrm{ml})(\mathrm{b})\end{array}$ & $\begin{array}{l}\text { Main effect } \\
(\mathrm{ml})(\mathrm{a}-\mathrm{b})\end{array}$ \\
\hline \multirow{10}{*}{$\begin{array}{l}\text { Known } \\
\text { combinations } \\
\text { and models }\end{array}$} & 1 & 0.65 & 8.11 & 8.27 & -0.16 & 38.89 & 10.38 & 10.24 & 0.14 \\
\hline & 15 & & & & & 11.11 & 10.04 & 10.32 & -0.29 \\
\hline & 21 & 8.39 & 9.45 & 8.16 & $1.30 * *$ & 11.11 & 10.38 & 10.28 & 0.10 \\
\hline & 26 & 4.52 & 9.40 & 8.21 & $1.19 *$ & & & & \\
\hline & 38 & 2.58 & 8.62 & 8.26 & 0.37 & 11.11 & 10.42 & 10.28 & 0.14 \\
\hline & 54 & 0.65 & 8.59 & 8.26 & 0.33 & 5.56 & 9.63 & 10.33 & -0.70 \\
\hline & LMW-2 & 14.19 & 9.07 & 8.13 & $0.94 * *$ & 77.78 & 10.28 & 10.33 & -0.05 \\
\hline & aaa & 12.90 & 9.04 & 8.15 & $0.89 *$ & 61.11 & 10.39 & 10.15 & 0.24 \\
\hline & dab & 1.29 & 9.41 & 8.25 & 1.16 & 16.67 & 9.90 & 10.37 & -0.47 \\
\hline & LMW-2 ${ }^{-}$ & 6.45 & 9.33 & 8.19 & $1.14 *$ & & & & \\
\hline \multirow{3}{*}{$\begin{array}{l}\text { Unreported } \\
\text { combinations }\end{array}$} & 45 & 0.65 & 11.56 & 8.24 & $3.31 *$ & & & & \\
\hline & 95 & 2.58 & 10.53 & 8.20 & $2.33 * *$ & & & & \\
\hline & 123 & 0.65 & 11.68 & 8.24 & $3.44 *$ & & & & \\
\hline
\end{tabular}

* $P<0.05$; ** $P<0.01 ; * * * P<0.001$

a SDS value of the landraces carrying the combination

given model were recorded in Algerian (83.3 \%) LR and MC (77.8\%), with the exclusive presence of model LMW-2. LR from Italy, Tunisia and Morocco had both models, at frequencies ranging from 54 to $60 \%$. Model LMW-2- $2^{-}$was missing in three of the countries clustered in branch B (Jordan, Portugal and Lebanon). Both models were present in Spanish and Turkish LR, at a frequency of 14 and $27 \%$, respectively. The mean SDS-sedimentation values of the genotypes included in each cluster branch were 9.2, 8.2 and 8.0 for branches A, B and C, respectively.

\section{Discussion}

Results of a previous study involving SSR-based molecular diversity evaluation indicated that the 155 LR used here may be considered as representative of the genetic diversity of ancient local durum populations from the Mediterranean Basin. Nazco et al. (2012) reported large variability for yield and quality traits in the same set of LR used here, which were useful to separate three major geographical regions within the Mediterranean Basin (West, East and North Balkan) with contrasting qualitative and productive characteristics. The same study concluded that the
Eastern Mediterranean LR retained the greatest variability, which is consistent with this region being the geographic center of genetic diversity for wheat (Feldman 2001). Although the number of MC included in this study was much smaller than that of LR, they were a representative set of the currently commercially grown durum germplasm in the Mediterranean Basin. It is therefore reasonable to assume that the alleles or banding patterns that would be present in the overall modern Mediterranean germplasm are likely to be represented within the group of $18 \mathrm{MC}$ included in this research. In a previous study (Royo et al. 2010), conducted with a collection of 191 accessions representing the existing genetic diversity within the same geographical area, the same MC were found to represent distinct genetic groups, according to their genetic structure assessed with SSR markers. In that study, the subpopulation related to the CIMMYT hallmark founder 'Altar 84', whose derivatives have been largely grown under different names in North Africa, included cultivars such as 'Gallareta', 'Sula' and 'Astigi'. On the other hand, 'Vitron' and 'Meridiano' belonged to a different group genetically close to the CIMMYT founder 'Yavaros 79', also released with different names in several Mediterranean countries. 'Simeto' was found to be part of an 







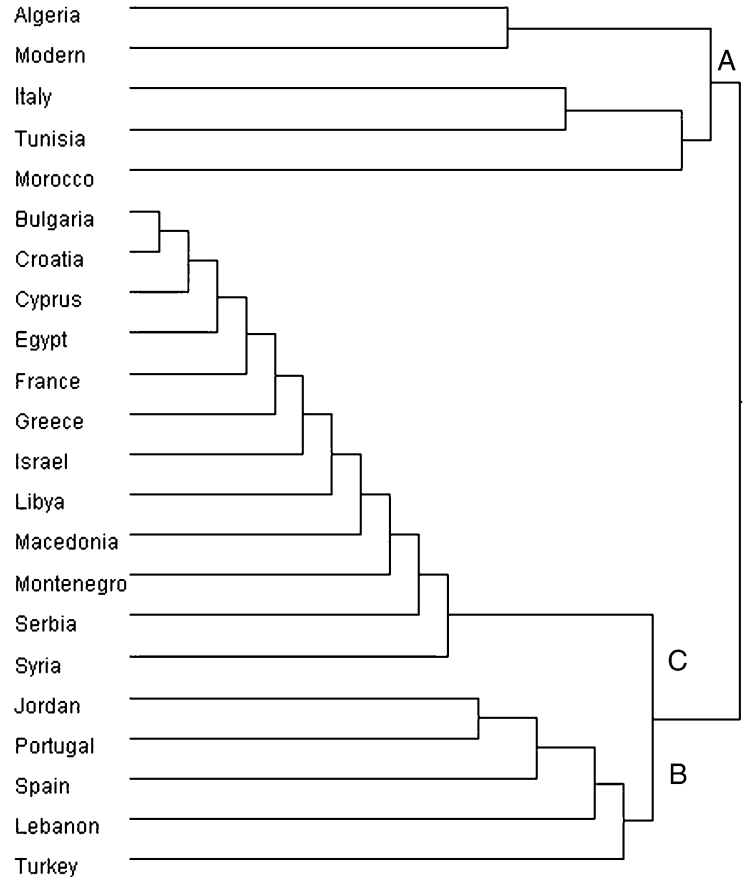

Fig. 1 Cluster analysis based on LMW combinations frequency in MC and by country for landraces

Italian genetic pool formed by genotypes derived from the founder cultivar 'Valnova', and 'Boabdil' was assigned within the more recently formed Spanish genetic pool (Royo et al. 2010). In the same study cultivars 'Claudio', 'Svevo', 'Senadur' and 'Bolo' could not be assigned to any structured subpopulation.

A purification process was required to evaluate material with a minimum homogeneity in their quality performance and glutenin allelic composition. This may have resulted in the decrease of intra-LR variability but is not considered an issue in the present study given the fact that its primary focus was the diversity among, not within, LR.

The detection of 131 different allelic/banding pattern combinations recorded in the present germplasm collection, representing $76 \%$ of the 173 theoretical possible haplotype combinations, reveals large genetic variability for glutenin composition. However, there were substantial differences between countries. Although the number of entries varied widely between countries, our results are in accordance with the conclusions of previous studies reporting larger genetic diversity in LR from the Iberian Peninsula (Moragues et al. 2006c), and lower variability in Bulgarian and Algerian representatives (Moragues et al. 2006c; Hamdi et al. 2010). Thus, low levels of genetic diversity may have resulted from a process of centuries of selection by farmers in response to adaptation to specific environmental conditions and/or users preferences.

The 32 combinations found at HMW-GS loci were based on the joint expression of 5 and 20 allelic variants at Glu-Al and Glu-B1 loci, respectively, while the 98 combinations detected at LMW-GS loci were related to the expression of 15 alleles or production of banding patterns at Glu-A3 locus, 72 at Glu-B3 and 2 at Glu-B2 (Nazco et al. 2013). This diversity is substantially greater than reported by previous studies conducted in durum wheat (Turchetta et al. 1995; Cherdouh et al. 2005; Moragues et al. 2006c). The considerable variability of glutenin combinations found in LR can potentially enhance their value in breeding to broaden the genetic basis of gluten quality improvement, beyond what can be achieved by using modern germplasm. These may be particularly useful to improve gluten strength, or to produce gluten properties for specific products.

The alleles expressed by loci producing LMW-GS in the present germplasm group produced only two of the five LMW models described by Nieto-Taladriz et al. (1997), with the notable absence of the LMW-1related models, which are known to be associated with very weak gluten properties. The diversity found for the LMW-GS loci in the present group of germplasm is consequently narrower than that observed in previous studies conducted with other Mediterranean LR (Carrillo et al. 1990; Moragues et al. 2006c), which did not include representatives from Croatia, France, Israel, Jordan, Libya, Macedonia, Montenegro and Serbia all countries with LR characterized by limited diversity in LMW models. Model LMW-2 (aaa) was frequent in LR from Lebanon, Turkey and Jordan, suggesting a putative location of origin of this combination. On the other hand, model LMW-2 (dab) was detected in two Spanish LR ('Recio de Almería' and 'Rubio de Miajadas'), and not in LR from countries from which the Spanish germplasm may have originated, hinting at possible post-migration mutation (once established in the Iberian Peninsula) followed by local selection.

In agreement with previous studies (Liu and Shepherd 1996; Cherdouh et al. 2005), cluster analysis grouped MC with genotypes from Italy and the three 
Maghreb countries due to the high frequency of LMW models type 2. A second group clustered countries in which LMW-2 models were found from medium to low frequencies (Turkey, Jordan, Lebanon, Portugal and Spain), while a third branch joined 12 countries in which LMW-2 models were absent. Our results associated the presence of LMW-2 with strong gluten, in agreement with previous studies showing the favorable effects of LMW-2 on durum wheat quality (Pogna et al. 1990; Masci et al. 2000; Raciti et al. 2003).

In the present collection, LMW type 1 models, known to be associated with very weak gluten properties (Pogna et al. 1990; Masci et al. 2000; Raciti et al. 2003; Peña and Pfeiffer 2005), were most notably absent. This is of high relevance both from an evolutionary standpoint as well as from the practical perspective of identifying combinations with strengthenhancing effects. In relation to evolution, it is well known that LMW type 1 models are indeed present in numerous genetic resources as well as MC from the Mediterranean region, some very important and still widely grown (Carrillo et al. 1990; Moragues et al. 2006c). Yet, by chance alone, our selection of the present collection of 155 LR based on geographical origin and SSR-detected diversity (by enlarge neutral and presumably not discriminating against any particular allele) did not include any genotype with allelic combinations resulting in such model. This suggests that selection against LWM type 1 models, possibly because of their association with weak gluten properties, may have started and was implemented by local farmers/users much before it was consciously applied, based on scientific findings, by modern wheat breeders in more modern germplasm in response to the industrialization of the pasta, cous-cous and even bread industries.

The absence of LMW type 1 models in the present set of germplasm is also important as it provides an opportunity to detect relatively smaller allelic/banding patterns strength-enhancing effects without the contrast LMW type 1 versus LMW type 2 which results in considerable strength differences, often masking or making undetectable smaller differences associated with other alleles/banding patterns, some of which could be used to our advantage in breeding. In this context, the present study enabled us to detect various interesting trends and effects. The combination LMW-2 (aaa) with the null allele at Glu-Al locus and the $7+8$ at Glu-Bl (number 1) was the most frequent (39\%) in MC. The results of the Fisher's exact test confirmed that differences between gluten strength groups were associated with the frequency of this combination, supporting the conclusions of previous studies identifying it's suitability for high gluten strength improvement (Raciti et al. 2003; Sissons et al. 2005).

On the other hand, our results were not in agreement with previous studies (Carrillo et al. 1990; Raciti et al. 2003; Sissons et al. 2005) regarding the negative effect on gluten strength associated with the presence of band 20 at Glu-B1. In the present study, three of the combinations resulting in a significant enhancement of gluten strength (numbers 21, 26 and 95) had band 20 at Glu-B1, and for combinations 21 and 95 differences between gluten strength groups were even associated with the allelic/banding pattern combination frequency. It can be hypothesized that on these three combinations the putative negative effect of band 20 was compensated by the positive effect of banding pattern $2+4+15+19$ at Glu-B3 as reported by Nieto-Taladriz et al. (1997). These results stress the fact that frequently the interactions between HMWGS and LMW-GS in the co-formation of the gluten complex is more important than main effects of a single allele or banding pattern (Ruiz and Carrillo 1995). The discrepancies between studies addressing the effect of alleles at Glu-Bl locus on grain quality led some authors to avoid the use of allelic variation at this locus as a basis in selecting for gluten strength in durum wheat (Sissons 2008). Moreover, the effect of the alleles at $G l u-B 1$ locus seems also to depend on the allelic pattern at Glu-B3 (Martinez et al. 2005).

The lack of significant effect on gluten strength associated with any of the combinations identified in MC was probably due to the fixing of this trait at high levels (SDS-sedimentation test values >9) through selection. Among the LR there were only two known combinations that were associated with a strengthenhancing effect. Their presence increased the SDSsedimentation test values by about $14.6 \%$ (from 8.2 to 9.4). The first of these (number 21: null allele at Glu$A 1$ locus, band 20 at Glu-B1 locus and bands 6, $2+4+15+19$ and 12 at Glu-A3, Glu-B3 and Glu$B 2$ loci, respectively), which included the LMW-2 (aaa) model, was present in $11.1 \%$ of $\mathrm{MC}$ and about $8.4 \%$ of LR and old Italian cultivars. The second (number 26, differing from the previous one only in 
the presence of the null allele at Glu-A3 locus), which included the LMW-2- (haa) model, was absent in MC and was found in 7 LR (4.5\%). However, our data did not confirm that differences between gluten strength groups were associated with the frequency of this combination within each group. Despite the presence of LMW-2 (dab) was associated with an increase in SDS-sedimentation value of about $14 \%$, its effect was not statistically significant, probably due to the fact that it was detected only in two genotypes, both Spanish LR.

This study identified LMW-2 (aaa) in three modern Italian cultivars ('Simeto', 'Meridiano' and 'Claudio'), and LMW-2 (dab) in 'Svevo', another modern Italian cultivar. The presence of LMW-2 (aaa) in 'Simeto' and 'Meridiano' was probably inherited from the North African LR 'Jennah Khortifa', via the landmark Italian cultivar 'Senatore Capelli' (released in 1915). This cultivar, also known as 'Capelli', is an ancestor of many Italian cultivars, some of them included in this study and identified as having LMW-2 (aaa), such as 'Capeiti 8' (derived from the cross Senatore Capelli/Eiti), and the MC 'Simeto' (Capeiti 8/Valnova) and 'Meridiano' (Simeto/Wb881/Duilio// F21). Accordingly, LMW-2 ${ }^{-}$(haa) in 'Trinakria' (derived from the cross B14/Capeiti 8) could not come from 'Capeiti 8', but probably from the parent line B14. LMW-2 (aaa) in 'Claudio' (CIMMYT selection/Durango//ISI938/Grazia) and LMW-2 (dab) in 'Svevo' (CIMMYT selection/Zenit) may have been contributed from the CIMMYT lines used as parents.

Our results showed that $78 \%$ of the MC in this study and including those with outstanding gluten strength ('Ocotillo', 'Claudio' and 'Meridiano') had the LMW-2 model, indicating the extensive selection by breeding programs of this gluten-strength enhancing combination. LMW-2 was absent in only four MC, one with high gluten strength (the French cultivar 'Arment'), and three with very high gluten strength, namely the Spanish cultivars 'Ancalei', 'Astigi' and 'Boabdil', all three derived from or involving CIMMYT germplasm. This indicates that no allele, no matter how strength-enhancing, is absolutely necessary for adequate strength and therefore, improvement of this trait can be achieved by different breeding strategies using alternative alleles or allelic combinations, provided a minimum of information on their strength-enhancing effect is reliably generated.
This study identified three novel combinations that were associated with significant increase in the gluten strength of the LR carrying them. Combination number 45 (null allele and band $6+8$ at Glu-Al and Glu-B1 loci, respectively, and bands 11, $2+4+15+18+19$ and the null allele at Glu$A 3, G l u-B 3$ and Glu-B2 loci, respectively) was identified in the French LR 'Trigo Glutinoso' (PI-174699), which was reported in a previous study as having high EU quality index and a high sedimentation index calculated as the quotient between gluten strength and protein content (Nazco et al. 2012). A second combination (number 123), formed by bands $2^{*}$ and $7+17$ at Glu-Al and Glu-BI loci, respectively and bands $6,2+4+14+15+18$ and the null allele at Glu-A3, Glu-B3 and Glu-B2 loci, respectively, was present in the Egyptian LR identified in the USDA gene bank as 'PI-366109', which reached the highest SDS-sedimentation test value of all the genotypes included in this study $(11.7 \mathrm{ml})$. This LR had been previously reported to have very high EU quality index, protein content and sedimentation index (Nazco et al. 2012). It is important to note that this particular genotype had 1 of the 54 combinations ( $41 \%$ of the total 131 combinations) with a non-null allele at GluAl but was one of the very few with a significantly enhanced strength. While non-null alleles at this locus have been strongly associated with increased gluten strength in bread wheat (Payne et al. 1987), they do not seem to have the same generalized effect in durum wheat. Rather, they appear to be associated with enhanced strength only when interacting with other sub-units, in relatively rare cases. The positive effect of combination 95 (bands 1 and 20 at Glu-Al and Glu$B 1$ loci, respectively, and bands $6+20$, $2+4+15+19$ and 12 at Glu-A3, Glu-B3 and Glu-B2 loci, respectively) was based on the results obtained in four Turkish LR. Among them genotypes BGE-019265 and BGE-019264 seem to be the most interesting for breeding purposes due to their outstanding gluten strength.

In the present study, gluten strength was measured on samples originating from a single location. While some environmental effect on the expression of gluten strength was taken into account through the analyses of data from three growing seasons, the results obtained herein may be extrapolated to other environments only with some caution. However, gluten strength, especially as determined by the SDS- 
sedimentation test, is known to be a highly inheritable trait, controlled by genotype to a much greater extent than by the environment (Dencic et al. 2011; Pecetti and Annicchiarico 1993). In fact, of the most important quality traits in durum wheat such as grain yellow color, kernel characteristics, protein or ash content, gluten strength is the least affected by the environment. Based on this fact, it is reasonable to expect that the medium to large differences between the different glutenin combinations observed in this study may extend to other environments. No inference can be made with regards to small differences between glutenin combinations.

Finally, it is important to note that the scope of the present study is limited to relating glutenin composition to an estimate of gluten strength, a single of several quality attributes important to end-product overall quality. A given glutenin composition of the many identified exclusively in the LR (not present in the MC) can be considered positive or negative or neutral with regards to gluten strength but without making any inferences on its impact on overall end-product quality, as the latter is highly variable depending on the endproduct itself, the transformation process and cooking/ preparation protocols and cannot be addressed by different levels of gluten strength alone.

Acknowledgments R. Nazco was recipient of a PhD grant from the Comisionat per Universitats i Investigació del Departament d'Innovació, Universitats i Empresa of the Generalitat de Catalunya and the Fondo Social Europeo. This study was partially funded by CICYT under projects AGL200911187 and AGL-2012-37217 and was developed within the framework of the agreement between INIA Spain and CIMMYT. The Centre UdL-IRTA is part of the Centre CONSOLIDER INGENIO 2010 on Agrigenomics funded by the Spanish Ministry of Education and Science. Thanks are given to CRF-INIA, ICARDA and USDA Germplasm Bank for providing accessions for this study.

Open Access This article is distributed under the terms of the Creative Commons Attribution License which permits any use, distribution, and reproduction in any medium, provided the original author(s) and the source are credited.

\section{References}

Axford DWE, McDemott EE, Redman DG (1978) Small scale test of bread making quality. Milling Feed Fert 161:18-20

Boggini G, Pogna NE (1989) The bread making quality and storage protein composition of Italian durum wheat. J Cereal Sci 9:131-138
Camacho Villa TC, Maxted N, Scholten MA, Ford-Lloyd BV (2005) Defining and identifying crop landraces. Plant Genet Res 3:373-384

Carrillo JM, Vázquez JF, Orellana J (1990) Relationship between gluten strength and glutenin proteins in durum wheat cultivars. Plant Breed 104:325-333

Cherdouh A, Khelifi D, Carrillo JM, Nieto-Taladriz MT (2005) The high and low molecular weight glutenin subunit polymorphism of Algerian durum wheat landraces and old cultivars. Plant Breed 124:338-342

Dencic S, Mladenov N, Kobiljski B (2011) Effects of genotype and environment on breadmaking quality in wheat. Int $\mathrm{J}$ Plant Prod 5:71-81

DuCros DL (1987) Glutenin proteins and gluten strength in durum wheat. J Cereal Sci 5:3-12

Feldman M (2001) Origin of cultivated wheat. In: Bonjean AP, Angus WJ (eds) The world wheat book. A history of wheat breeding. Lavoisier Publishing, Paris, pp 3-56

Hamdi W, Bellil I, Branlard G, Khelifi D (2010) Genetic variation and geographical diversity for seed storage proteins of seventeen durum wheat populations collected in Algeria. Not Bot Horti Agrobot Cluj 38:22-32

Liu CY (1995) Identification of a new low $M_{r}$ glutenin subunit locus on chromosome 1B of durum wheat. J Cereal Sci 21:209-213

Liu CY, Shepherd KW (1996) Variation of B subunits of glutenin in durum, wild and less-widely cultivated tetraploid wheats. Plant Breed 115:172-178

MacKey J (2005) Wheat: its concept, evolution, and taxonomy. In: Royo C, Nachit MM, Di Fonzo N, Araus JL, Pfeiffer WH, Slafer GA (eds) Durum wheat breeding: current approaches and future strategies. Food Products Press, New York, pp 3-61

Martinez MC, Ruiz M, Carrillo JM (2004) New B low $\mathrm{M}_{\mathrm{r}}$ glutenin subunit alleles at the Glu-A3, Glu-B2 and Glu-B3 loci and their relationship with gluten strength in durum wheat. J Cereal Sci 40:101-107

Martinez MC, Ruiz M, Carrillo JM (2005) Effects of different prolamin alleles on durum wheat quality properties. J Cereal Sci 41:123-131

Masci S, D’Ovidio R, Lafiandra D (2000) A 1B-coded lowmolecular-weight glutenin subunit associated with quality in durum wheats shows strong similarity to a subunit present in some bread wheat cultivars. Theor Appl Genet 100:396-400

Moragues M, García del Moral LF, Moralejo M, Royo C (2006a) Yield formation strategies of durum wheat landraces with distinct pattern of dispersal within the Mediterranean basin: I. Yield components. Field Crops Res 95:194-205

Moragues M, García del Moral LF, Moralejo M, Royo C (2006b) Yield formation strategies of durum wheat landraces with distinct pattern of dispersal within the Mediterranean basin: II. Biomass production and allocation. Field Crops Res 95:182-193

Moragues M, Zarco-Hernández J, Moralejo MA, Royo C (2006c) Genetic diversity of glutenin protein subunits composition in durum wheat landraces [Triticum turgidum ssp. turgidum convar. durum (Desf.) MacKey] from the Mediterranean Basin. Genet Resour Crop Evol 53:993-1002 
Moragues M, Moralejo M, Sorrells ME, Royo C (2007) Dispersal of durum wheat landraces across the Mediterranean basin assessed by AFLPs and microsatellites. Genet Resour Crop Evol 54:1133-1144

Nazco R, Villegas D, Ammar K, Peña RJ, Moragues M, Royo C (2012) Can Mediterranean durum wheat landraces contribute to improved grain quality attributes in modern varieties? Euphytica 185:1-17

Nazco R, Peña RJ, Ammar K, Villegas D, Crossa J, Moragues M, Royo C (2013) Variability in glutenin subunit composition of Mediterranean durum wheat germplasm and its relationship with gluten strength. J Agric Sci Camb. doi:10. 1017/S0021859613000117

Nevo E, Payne PI (1987) Wheat storage proteins: diversity of HMW glutenin subunits in wild emmer from Israel. 1. Geographical patterns and ecological predictability. Theor Appl Genet 74:827-836

Nieto-Taladriz MT, Ruiz M, Martínez MC, Vázquez JF, Carrillo JM (1997) Variation and classification of B low-molecularweight glutenin subunit alleles in durum wheat. Theor Appl Genet 95:1155-1160

Payne PI, Jackson EA, Holt LM (1984) The association between $\gamma$-gliadin 45 and gluten strength in durum wheat varieties: a direct causal effect or the result of genetic linkage? J Cereal Sci 2:73-81

Payne PI, Nightingale MA, Krattiger AF, Holt LM (1987) The relationship between HMW glutenin subunit composition and the bread-making quality of British-grown wheat varieties. J Sci Food Agric 40:51-65

Pecetti L, Annicchiarico P (1993) Grain-yield and quality of durum-wheat landraces in a dry Mediterranean region of Northern Syria. Plant Breed 110:243-249

Peña RJ, Pfeiffer WH (2005) Breeding methodologies and strategies for durum wheat quality improvement. In: Royo C, Nachit MM, Di Fonzo N, Araus JL, Pfeiffer WH, Slafer GA (eds) Durum wheat breeding: current approaches and future strategies. Food Products Press, New York, pp 663-702

Peña RJ, Amaya A, Rajaram S, Mujeeb-Kazi A (1990) Variation in quality characteristics associated with some spring $1 \mathrm{~B} /$ 1R translocation wheats. J Cereal Sci 12:105-112

Peña RJ, Gonzalez-Santoyo H, Cervantes F (2004) Relationship between Glu-D1/Glu-B3 allelic combinations and breadmaking quality-related parameters commonly used in wheat breeding. In: Masci S, Lafiandra D (eds) Proceedings 8th gluten workshop. RACI, Australia, pp 156-157

Pogna NE, Autran JC, Mellini F, Lafiandra D, Feillet P (1990) Chromosome 1B-encoded gliadins and glutenin subunits in durum wheat: genetics and relationship to gluten strength. J Cereal Sci 11:15-34
Raciti CN, Doust MA, Lombardo GM, Boggini G, Pecetti L (2003) Characterization of durum wheat Mediterranean germplasm for high and low molecular weight glutenin subunits in relation with quality. Eur J Agron 19:373-382

Royo C, Elias EM, Manthey FA (2009) Durum wheat breeding. In: Carena MJ (ed) Handbook of plant breeding: cereals. Springer, Berlin, pp 199-226

Royo C, Maccaferri M, Álvaro F, Moragues M, Sanguineti MC, Tuberosa R, Maalouf F, García del Moral LF, Demontis A, Rhouma S, Nachit M, Nserallah N, Villegas D (2010) Understanding the relationships between genetic and phenotypic structures of a collection of elite durum wheat accessions. Field Crops Res 119:91-105

Ruiz M, Carrillo JM (1993) Linkage relationships between prolamin genes on chromosomes $1 \mathrm{~A}$ and $1 \mathrm{~B}$ of durum wheat. Theor Appl Genet 87:353-360

Ruiz M, Carrillo JM (1995) Relationship between different prolamin proteins and some quality parameters in durum wheat. Plant Breed 114:40-44

SAS Institute Inc. (2009) SAS/STAT ${ }^{\circledR}$ 9.2. user's Guide, 2nd edn. SAS Institute Inc., Cary

Shewry PR, Halford NG, Tatham AS (1992) High molecular weight subunits of wheat glutenin. J Cereal Sci 15:105-120

Singh NK, Shepherd KW (1988) Linkage mapping of genes controlling endosperm storage proteins in wheat. 2. Genes on the long arms of group 1 chromosomes. Theor Appl Genet 75:642-650

Sissons M (2008) Role of durum wheat composition of the quality of pasta and bread. Food 2:75-90

Sissons MJ, Ames NP, Hare RA, Clarke JM (2005) Relationship between glutenin subunit composite on and gluten strength measurements in durum wheat. J Sci Food Agric $85: 2445-2452$

Tarekegne A, Labuschagne MT (2005) Relationship between high molecular weight glutenin subunit composition and gluten quality in Ethiopian grown bread and durum wheat cultivars and lines. J Agron Crop Sci 191:300-307

Turchetta T, Ciaffi M, Porceddu E, Lafiandra D (1995) Relationship between electrophoretic pattern of storage proteins and gluten strength in durum wheat landraces from Turkey. Plant Breed 114:406-412

Vázquez JF, Ruiz M, Nieto-Taladriz MT, Albuquerque M (1996) Effects of gluten strength of low Mr glutenin subunits coded by alleles at the Gli-A3 and Glu-B3 loci in durum wheat. J Cereal Sci 24:125-130

Weir BS (1996) Genetic data analysis II. Sinauer Associates Inc., Sunderland 